\title{
The validity of the kinetic collection equation revisited - Part 2: Simulations for the hydrodynamic kernel
}

\author{
L. Alfonso ${ }^{1}$, G. B. Raga ${ }^{2}$, and D. Baumgardner ${ }^{2}$ \\ ${ }^{1}$ Universidad Autónoma de la Ciudad de México, México City, 09790 México \\ ${ }^{2}$ Centro de Ciencias de la Atmósfera, Universidad Nacional Autónoma de México, México City, 04510 México
}

Received: 19 January 2010 - Published in Atmos. Chem. Phys. Discuss.: 5 March 2010

Revised: 7 July 2010 - Accepted: 7 July 2010 - Published: 5 August 2010

\begin{abstract}
The kinetic collection equation (KCE) has been widely used to describe the evolution of the average droplet spectrum due to the collection process that leads to the development of precipitation in warm clouds. This deterministic, integro-differential equation only has analytic solution for very simple kernels. For more realistic kernels, the KCE needs to be integrated numerically. In this study, the validity time of the KCE for the hydrodynamic kernel is estimated by a direct comparison of Monte Carlo simulations with numerical solutions of the KCE. The simulation results show that when the largest droplet becomes separated from the smooth spectrum, the total mass calculated from the numerical solution of the $\mathrm{KCE}$ is not conserved and, thus, the KCE is no longer valid. This result confirms the fact that for kernels appropriate for precipitation development within warm clouds, the KCE can only be applied to the continuous portion of the mass distribution.
\end{abstract}

\section{Introduction}

One of the most important mechanisms for the formation of rain is the collision and coalescence of smaller droplets into larger ones. This process can be described by the integrodifferential kinetic collection equation (KCE) or stochastic collection equation, which in discrete form is expressed as (Pruppacher and Klett, 1997):

$$
\begin{aligned}
& \frac{\partial N(i, t)}{\partial t}= \\
& \frac{1}{2} \sum_{j=1}^{i-1} K(i-j, j) N(i-j) N(j)-N(i) \sum_{j=1}^{\infty} K(i, j) N(j)
\end{aligned}
$$

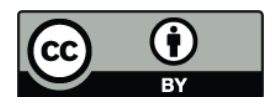

Correspondence to: L. Alfonso (lesterson@yahoo.com) where $N(i, t)$ is the total number of droplets with mass $x_{i}$ as a function of time. In Eq. (1), the time rate of change of the average number of droplets with mass $x_{i}$ is determined as the difference between two terms: the first term describes the average rate of production of droplets of mass $x_{i}$ due to coalescence between pairs of drops whose masses add up to mass $x_{i}$, and the second term describes the average rate of depletion of droplets with mass $x_{i}$ due to their collisions and coalescence with other droplets.

In real life, the collision-coalescence is a stochastic process but the KCE is a deterministic equation. For several successive realizations of the stochastic collection process (given set of probabilities and an initial distribution $N(i, 0)$ ), we would expect to obtain slightly different outcomes for the resulting droplet spectra. However, since Eq. (1) is deterministic, it can produce only one distribution once the collection kernel and the initial distribution $N(i, 0)$ are specified, and its solution is an average spectrum.

The average spectrum obtained from Eq. (1) and the ensemble average obtained from different realizations of the stochastic collection process are different. Bayewitz et al. (1974) showed that the solution of the KCE and the expected values calculated from the stochastic equation are equal only if the covariances are omitted from the probabilistic model. Valioulis and List (1984) compared the numerical solution of the KCE with averages calculated from different realizations of the stochastic process obtained from a Monte Carlo simulation for Brownian diffusion, a fluid shear and a differential sedimentation collision kernel. They showed that the solution of KCE matched well the true stochastic averages from the Monte Carlo simulation provided that the total number of particles was large.

Furthermore, the KCE assumes that the number of droplets $N(i, t)$ of mass $x_{i}$, is a continuous variable. In the case of runaway growth, when a single droplet, much larger than the second largest droplet, becomes detached from the continuous part of the distribution, such assumption is no longer

Published by Copernicus Publications on behalf of the European Geosciences Union. 
valid. At some point in the evolution of the mass spectrum there is a transition from a continuous one to one with a continuous distribution plus a massive runaway droplet. After the runaway growth occurs, the total mass (refered to as the liquid water content) is no longer conserved by the KCE and the averages obtained from the deterministic differential Eq. (1) will differ from the expected values obtained from the stochastic collection process.

It is expected to obtain a runaway droplet if the kernel $K\left(x_{i}, x_{j}\right)$ increases sufficiently rapid with $x_{i}$ and $x_{j}$. In astrophysical applications was demonstrated that the runaway growth occurs if the two body coalescence kernel rises faster than linearly in the mass of the heavier particle (Malyshkin and Goldman, 2001).

Wetherill (1990) concluded that the KCE only models the continuous part of the spectrum. Since no such particle is taken into account by the KCE, some matter escapes from the system and the total mass calculated from the KCE decreases with time. Alfonso et al. (2008) simulated the evolution of a droplet spectrum assuming a kernel that was proportional to the product of the masses of the colliding droplets. The mass of the largest (runaway droplet) was estimated using a Monte Carlo algorithm, and compared with the runaway droplet mass obtained by subtracting the mass of the continuous spectrum (obtained from the analytical solution of the $\mathrm{KCE}$ ) from the initial mass of the system, resulting in very good agreement. As the largest droplet continues to grow by accretion of smaller droplets; the mass (or liquid water content) of the continuous spectrum predicted by the KCE further decreases.

Analytical expressions for the validity time of the KCE only exists for very simple kernels, such as constant, the sum of the masses, $B\left(x_{j}+x_{j}\right)$ the product of the masses $C\left(x_{i} \times x_{j}\right)$ and their combination (e.g. polynomials). There are no analytical solutions of the KCE when more realistic kernels are considered. As the KCE is generally taken as the governing equation to model stochastic growth in cloud models, it is very important to estimate the validity time of the KCE for kernels relevant to cloud physics.

In Alfonso et al. (2008), the numerical criteria suggested by Inaba et al. (1999), to calculate the validity time for the KCE was compared with the analytical results obtained by Drake (1972) and Tanaka and Nakazawa (1994), with good agreement. Inaba et al. (1999) proposed that the stochastic property of the system becomes distinct around the beginning of runaway growth. At that point, the ratio of the standard deviation for the largest particle mass over all the realizations to the averaged value from all the realizations, reaches a maximum.

The main goal of this study is to demonstrate that the numerical criteria suggested by Inaba et al. (1999) can be used to estimate the validity time of the KCE for kernels relevant for the development of precipitation within warm clouds, by comparing the numerical solutions of the KCE with results from Monte Carlo simulations.
The paper is organized as follows: the next section includes overview of previous results obtained for the product kernel. In Sect. 3, we validate the numerical algorithm to solve the KCE and compare the numerical solutions with analytical ones available in the literature. The validation of the numerical criteria suggested by Inaba et al. (1999) for the case of the hydrodynamic kernel is presented in Sect. 4. Finally, in Sect. 5 we discuss the results and possible implications for cloud physics modeling.

\section{An overview of previous results}

Drake (1972) calculated the analytical solutions of the KCE for polynomials of the form $K\left(x_{i}, x_{j}\right)=C\left(x_{i} \times x_{j}\right)$. The time evolution of the second moment (with respect to the droplet distribution), $M_{2}(t)$, is given by:

$M_{2}(t)=\frac{M_{2}\left(t_{0}\right)}{1-C M_{2}\left(t_{0}\right) t}$

For the discrete case $M_{2}$ is defined as:

$M_{2}(t)=\sum_{i=1}^{N_{d}} x_{i}^{2} N(i, t)$

From Eq. (2), it is clear that $M_{2}$ is undefined when

$\tau=\left[C M_{2}\left(t_{0}\right)\right]^{-1}$

The time $t=\tau$, when the deterministic KCE predicts a divergence of $M_{2}$ and a decrease of the first moment, $M_{1}$ (total mass or liquid water content) is called the gel point. The decrease of the total mass $M_{1}$ after the gel point for a kernel proportional to the product of the masses, $K\left(x_{i}, x_{j}\right)=$ $C\left(x_{i} \times x_{j}\right)$, is obtained from the analytical solution:

$N(i, t)=N_{0} \frac{(i T)^{i-1}}{i \Gamma(i+1)} \exp (-i T)$

$T=C N_{0} v_{0}^{2} t$

derived by Scott (1968) for a monodisperse initial condition. In Eq. $(5,6), N_{0}$ and $v_{0}$ correspond to the initial number and volume of droplets, respectively. We simulate a cloud volume equal to $1 \mathrm{~cm}^{3}$, containing 100 droplets $\left(N_{0}\right)$ of $14 \mu \mathrm{m}$ in radius $\left(v_{0}=1.1494 \times 10^{-8} \mathrm{~cm}^{3}\right)$. The value proposed by Long (1974) was assumed for $C=5.49 \times 10^{10} \mathrm{~cm}^{3} \mathrm{~g}^{-2} \mathrm{~s}^{-1}$, then Eq. (4) leads to $\tau=1379 \mathrm{~s}$ ( with $M_{2}\left(t_{0}\right)=x_{0}^{2} N_{0}$ ). This value of the breakdown time, can be compared with the time when the total mass (or liquid water content)

$M_{1}(t)=\sum_{i=1}^{\infty} x(i) N(i, t)$

with $N(i, t)$ calculated from $(5,6)$ starts to decrease. Figure 1 shows that the total mass is no longer conserved after $1300 \mathrm{~s}$, in agreement with the value of $\tau=1379 \mathrm{~s}$ obtained from Eq. (4). 


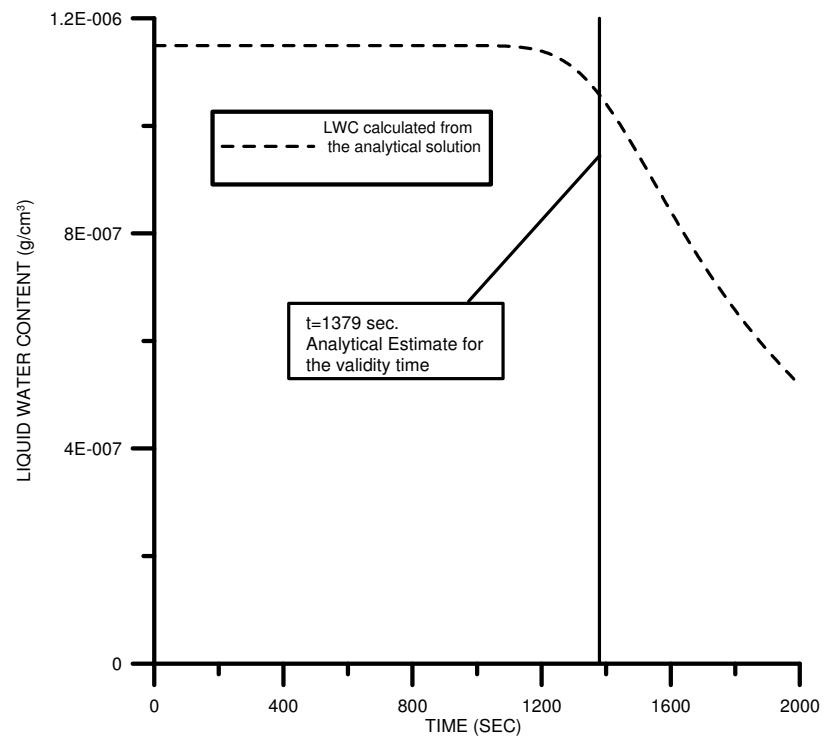

Fig. 1. Time evolution of the liquid water content (first moment of the distribution) for the product kernel $(K(x, y)=C x y$, $C=5.49 \times 10^{10} \mathrm{~cm}^{3} \mathrm{~g}^{-2} \mathrm{~s}^{-1}$ ), calculated from the analytical solution of the KCE.

The validity time obtained from (4) can be confronted with the estimation suggested by Inaba et al. (1999), and obtained by using a statistical code for modeling planetary accretion. They calculated the ratio of the standard deviation for the largest particle mass over all the realizations, to the average value evaluated from different realizations of the stochastic algorithm:

$M_{L 1, S}=\operatorname{STD}\left(M_{L 1}\right) / M_{L 1}$

The standard deviation $\left(\operatorname{STD}\left(\mathrm{M}_{L 1}\right)\right)$ for the mass of the largest droplet is calculated for each time by using the expression:

$\operatorname{STD}\left(M_{L 1}\right)=\sqrt{\frac{1}{N_{r}}\left(\sum_{i=1}^{N_{r}}\left(M_{L 1}^{i}-M_{L 1}\right)^{2}\right)}$

where $M_{L 1}$ is the ensemble mean of the mass of the largest droplet over all the realizations given by:

$M_{L 1}=\frac{1}{N_{r}} \sum_{i=1}^{N_{r}} M_{L 1}^{i}$

here $N_{r}$ is the number of realizations of the Monte Carlo algorithm and $M_{L 1}^{i}$ is the largest droplet for each realization. Inaba et al. (1999) found that $M_{L 1, S}$ was maximum in the vicinity of $M_{L 1} / M_{T}^{2 / 3}=1$ (where $M_{T}$ is the total mass of the system). This is consistent with the results of Tanaka and Nakazawa (1994), who demonstrated that for a kernel proportional to the product of the masses, the KCE is valid until the stage when a particle with a mass comparable to

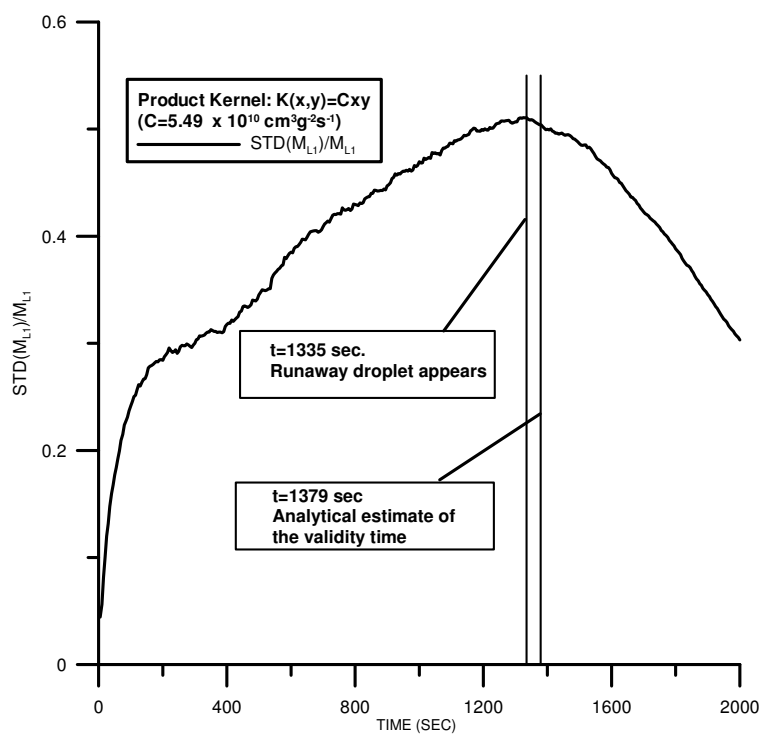

Fig. 2. The ratio (defined in Eq. 8) as a function of time, for the product kernel $K(x, y)=C x y\left(C=5.49 \times 10^{10} \mathrm{~cm}^{3} \mathrm{~g}^{-2} \mathrm{~s}^{-1}\right)$. Note that $\operatorname{STD}\left(M_{L 1}\right) / M_{L 1}$ reaches a maximum when the runaway droplet appears.

or larger than $M_{T}^{2 / 3}$ appears. As hypothesized by Inaba et al. (1999), this behavior of $\operatorname{STD}\left(M_{L 1}\right) / M_{L 1}$ indicates that the stochastic property becomes distinct when the runaway growth begins.

In Alfonso et al. (2008), the maximum of the statistics (8) was estimated from Monte Carlo simulations for the product kernel and compared with the values calculated from Eq. (4). The stochastic collection calculation was performed using the Monte Carlo method of Gillespie (1976), with the species accounting formalism proposed by Laurenzi et al. (2002). As can be observed in Fig. 2 , in the vicinity of $M_{L 1} / M_{T}^{2 / 3}=1$, the ratio $\operatorname{STD}\left(M_{L 1}\right) / M_{L 1}$ increases and reach a maximum at $\tau=1335 \mathrm{~s}$, very close to the analytical estimate $(1379 \mathrm{~s}) \mathrm{ob}$ tained from Eq. (4).

These arguments raise the question about the applicability of the statistics described by Eq. (8), to estimate the validity time for kernels relevant to cloud physics.

\section{Numerical integration of the $\mathrm{KCE}$ and comparison with analytical solutions}

In order to validate the statistics (8) for the hydrodynamic kernel, the KCE needs to be integrated numerically, and the solution obtained from the deterministic Eq. (1) compared with the averages calculated from the stochastic Monte Carlo algorithm. The numerical integration of Eq. (1) was performed using the Adams-Bashfort-Moulton predictorcorrector method (Alfonso et al., 2009). For the finite difference scheme, droplet mass in the numerical grid is expressed 


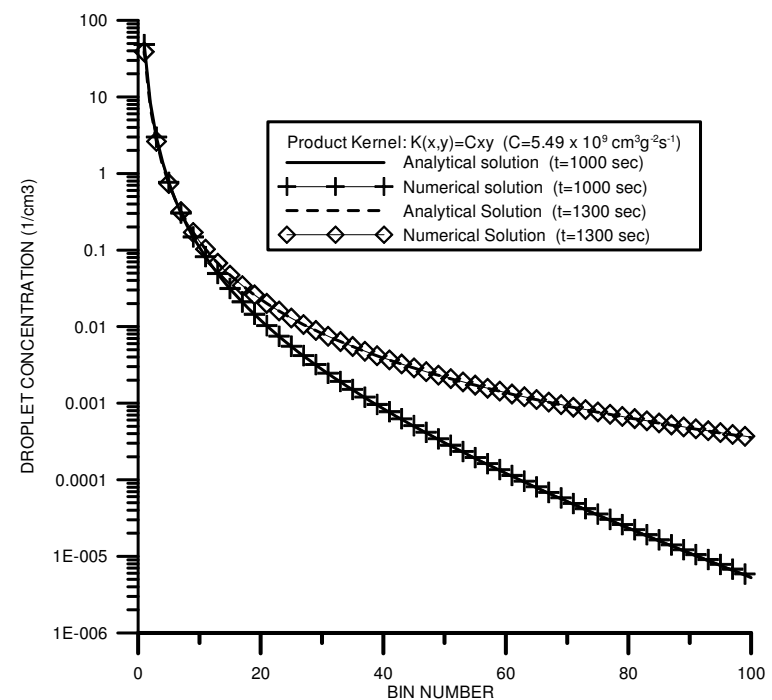

Fig. 3. Size distributions obtained from analytical solution of the $\mathrm{KCE}$ for the product kernel $K(x, y)=C x y$, $\left(C=5.49 \times 10^{10} \mathrm{~cm}^{3} \mathrm{~g}^{-2} \mathrm{~s}^{-1}\right)$, versus size distributions from numerical solution for two times $(t=1000,1300 \mathrm{~s})$.

as multiples of the mass of the initial $14 \mu \mathrm{m}$ monomer droplet. In order to check the performance of the finite difference method, the KCE was integrated numerically with the monodisperse initial condition $N(1,0)=100 \mathrm{~cm}^{-3}$ for the product kernel $K(x, y)=C x y \quad\left(C=5.49 \times 10^{10} \mathrm{~cm}^{3} \mathrm{~g}^{-2} \mathrm{~s}^{-1}\right)$, and the numerical results compared with the analytical solutions of the size distribution. Figure 3 shows the comparison of analytical and numerical solutions of the size distributions after 1000 and $1300 \mathrm{~s}$, highlighting the very good correspondence between them.

When gelation occurs, mass conservation is expected to break down in a finite time, there exists a $T_{g}$, called gelation time such that (which is equal to $\tau$ for the product kernel)

$$
\begin{aligned}
M_{1}(t) \equiv & M_{1}(0) \text { for } t<T_{g} \\
& \quad \text { and } \\
M_{1}(t)< & M_{1}(0) \text { for } t>T_{g}
\end{aligned}
$$

Simultaneously, a sudden growth of the second moment is to be expected (see Eq. 2). The first and second moments of the mass distribution were calculated from Eqs. (7) and (3), respectively, from the numerical solution of the KCE, to check whether the proposed numerical scheme provides a good estimate of the exact gelation time. Figure 4 displays the results for the time evolution of the liquid water content (first moment), indicating that it is no longer conserved after around $t=1300 \mathrm{~s}$, in agreement with the analytical value of the breakdown time obtained from Eq. (4). There is a sudden growth of the second moment near the breakdown time, confirming the expected "blow-up" behavior of $M_{2}(t)$ (see Eq. 2).

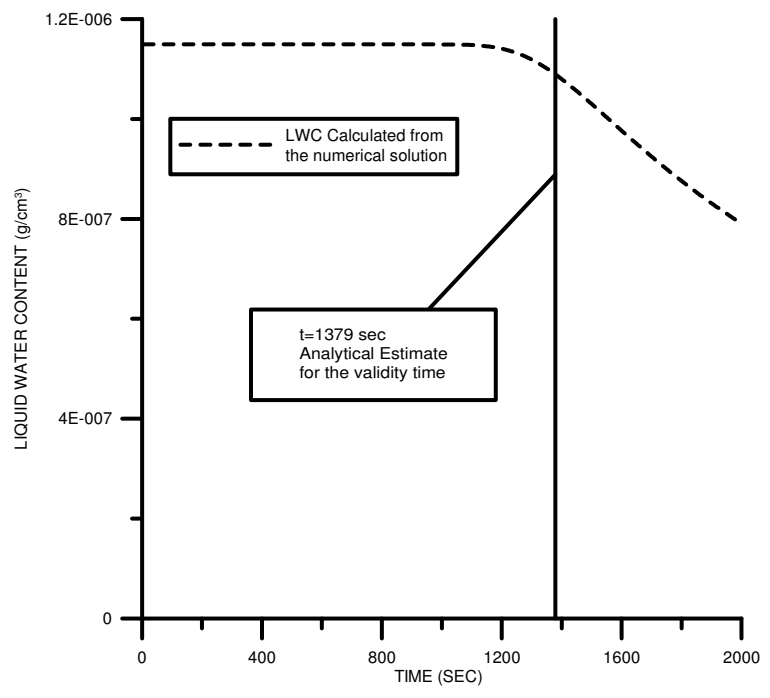

Fig. 4. Time evolution of the liquid water content (first moment of the distribution) for the product kernel $\left(K(x, y)=C x y, C=5.49 \times 10^{10} \mathrm{~cm}^{3} \mathrm{~g}^{-2} \mathrm{~s}^{-1}\right), \quad$ calculated from the numerical solution of the KCE.

\section{Estimation of the validity time of the KCE for the hydrodynamic kernel}

The development of precipitation in warm clouds is typically modeled by the collisions and coalescence between droplets, using a hydrodynamic kernel that takes into account the fact that droplets with different masses ( $m$ and $m^{\prime}$ and corresponding radii, $r$ and $r^{\prime}$ ) have different settling velocities, which are functions of their masses. Furthermore, droplets with different radii $\left(r\right.$ and $\left.r^{\prime}\right)$, will collide according with a varying efficiency of collision $\left(E\left(r, r^{\prime}\right)\right)$.Such hydrodynamic kernel has the form:

$K\left(m, m^{\prime}\right)=\pi\left(r+r^{\prime}\right)^{2}\left|V(m)-V\left(m^{\prime}\right)\right| E\left(r, r^{\prime}\right)$

where $V(m)$ and $V\left(m^{\prime}\right)$ are the terminal velocities of droplets with masses $m$ and $m^{\prime}$ respectively, and the values of the collision efficiencies $E\left(r, r^{\prime}\right)$ were taken from Hall (1980). These collision efficiencies are good for the purpose of our work, however, the modifications to Hall's collection efficiencies proposed in Kerkweg et al. (2003) can be considered in follow up studies.

In the first of two simulations performed for the hydrodynamic kernel we consider a system corresponding to a cloud volume of $1 \mathrm{~cm}^{3}$ and a bidisperse droplet distribution: 50 droplets of $14 \mu \mathrm{m}$ in radius, and another 50 droplets of $17.6 \mu \mathrm{m}$ in radius. We have calculated the behavior of the ratio $M_{L 1, S}$ (Eq. 8) evaluated from 1000 realizations of the Monte Carlo algorithm, and we have solved the KCE with a finite difference scheme to calculate the time evolution of the total liquid water content. Figure 6 shows that the liquid water content (or total mass) of the system from the integration 


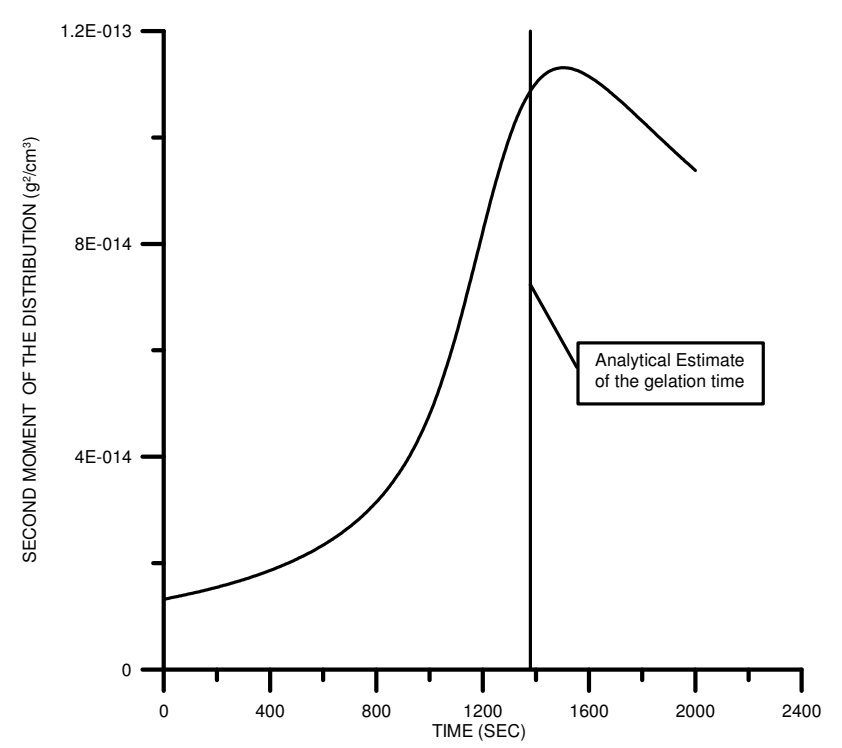

Fig. 5. Time evolution of the second moment of the distribution for the product kernel $(K(x, y)=C x y)$, calculated from the numerical solution of the KCE.

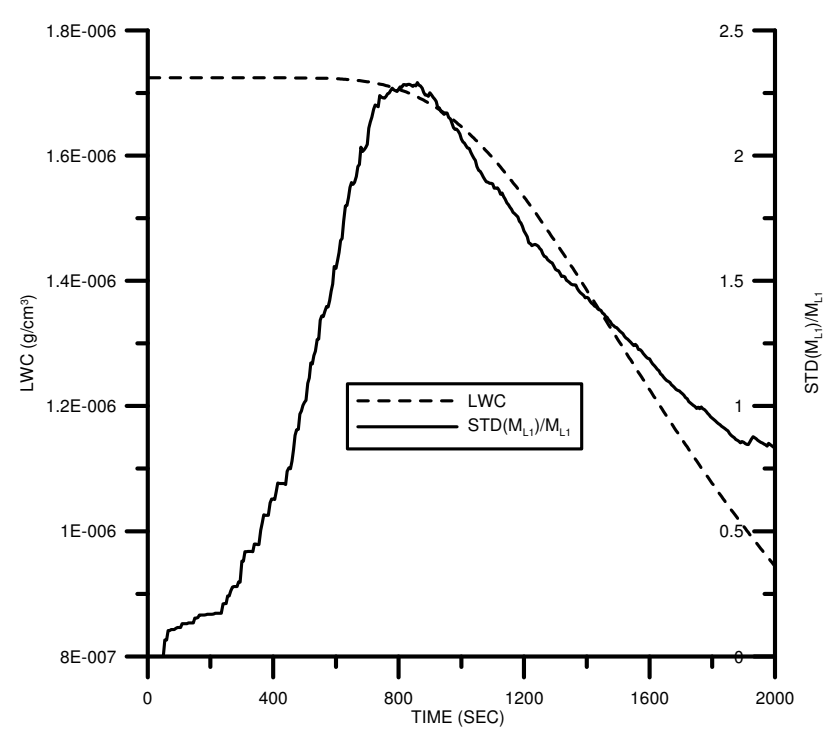

Fig. 6. Time evolution of total liquid water content calculated from the numerical solution of the KCE for the hydrodynamic kernel (dashed line) and the statistics $\operatorname{STD}\left(M_{L 1}\right) / M_{L 1}$ (solid line) estimated from the Monte Carlo algorithm. The simulations were performed for the hydrodynamic kernel with a bidisperse initial condition $N(1 ; 0)=50$ and $N(2 ; 0)=50$.

of the KCE is no longer conserved after $800 \mathrm{~s}$. This time is very close to the time when the statistics $M_{L 1, S}$ determined from the Monte Carlo realizations, reaches its maximum $(850 \mathrm{~s})$. This result confirms the fact that total mass calculated assuming a continuous droplet distribution starts

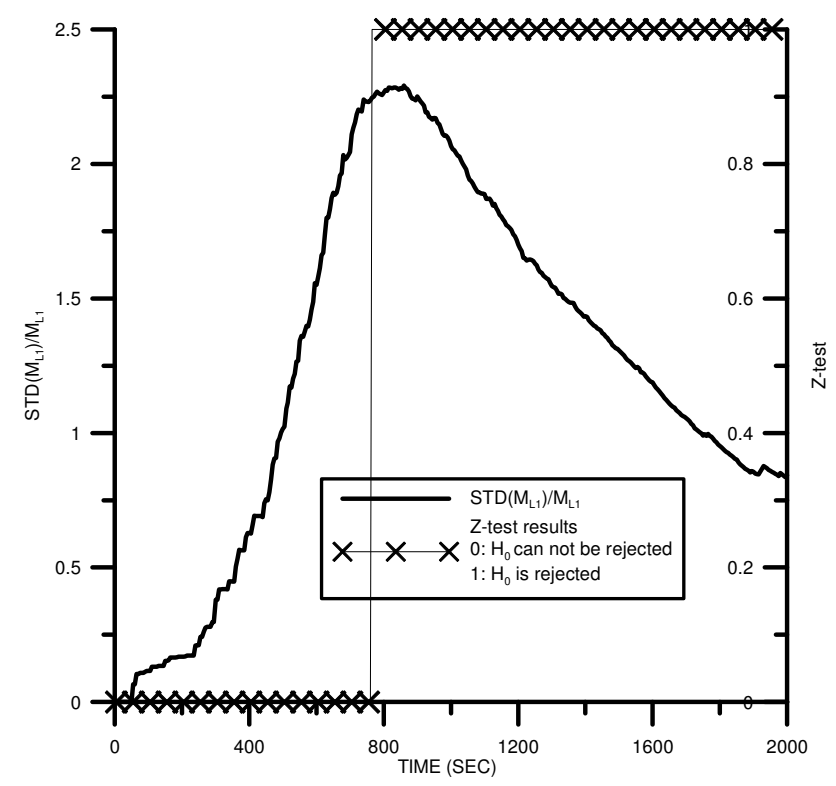

Fig. 7. Time evolution of the statistics $\operatorname{STD}\left(M_{L 1}\right) / M_{L 1}$ (thick solid line) estimated for 1000 realizations of the Monte Carlo algorithm and the results of the Z-test (thin solid line with crosses). The simulations were performed for the hydrodynamic kernel with a bidisperse initial condition $N(1 ; 0)=50$ and $N(2 ; 0)=50$.

to decrease around the time when the runaway droplet appears.

For the total droplet concentration, the Z-test was implemented to check whether the solution obtained from the deterministic KCE and the averages over 1000 realizations of the Monte Carlo method are equal. The null hypothesis would be $\mathrm{H}_{0}:\langle N\rangle=N$, where $\langle N\rangle$ is the true stochastic average calculated using the Monte Carlo method and $N$ is the average concentration calculated from the KCE. The results of the test of the hypothesis are displayed in Fig. 7. As expected, at a $5 \%$ significance level, the null hypothesis $\mathrm{H}_{0}$ : $\langle N\rangle=N$, is rejected after the time when the statistic $M_{L S, 1}$ reaches its maximum (around $800 \mathrm{~s}$ ).

In a second simulation, the initial number of droplets was set equal to $200(N(1 ; 0)=100$ and $N(2 ; 0)=100)$. The time evolution of the total liquid water content and the statistics (8) for this case are displayed in Fig. 8. Again there is a good correspondence between the time of the $M_{L 1, S}$ maximum $(430 \mathrm{~s})$ and the gelation time obtained from the numerical solution of the KCE (415 s).

The remarkable fact is that in Figs. 6 and 8, the 2 curves displayed in each figure were obtained independently from the numerical solution of the deterministic KCE (Eq. 1) and from the average over 1000 realizations of the Monte Carlo process, respectively. The results clearly indicate that the statistics (8) can be used as a good indicator of the validity time (gelation time) of the KCE when realistic kernels are used. This is an important issue since the KCE is generally 


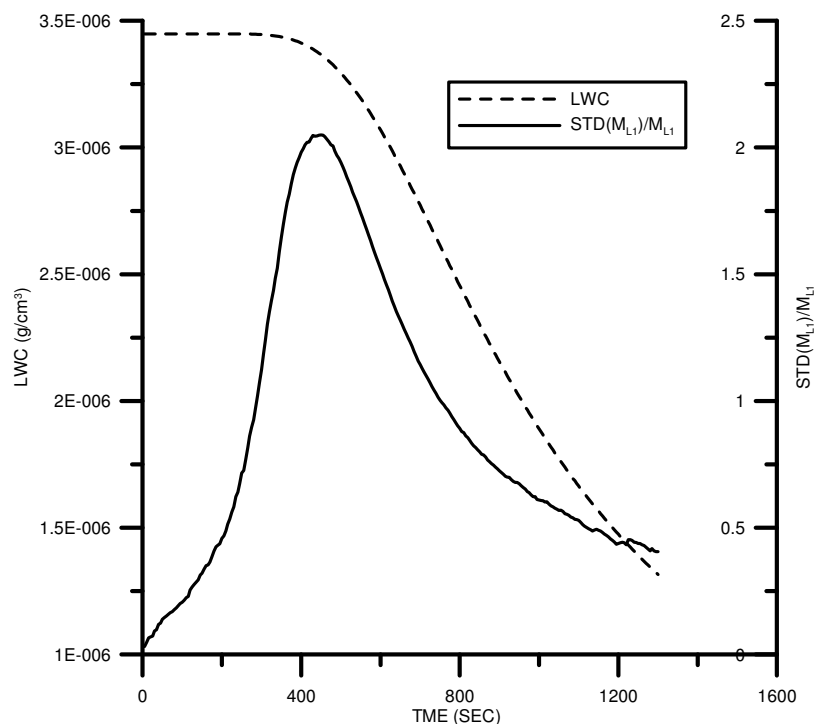

Fig. 8. Same as Fig. 6 but with the initial condition $N(1 ; 0)=100$ and $N(2 ; 0)=100$.

considered as the governing equation for stochastic collection growth. The results displayed in Figs. 6 and 8 also confirm the breakdown of the KCE for the hydrodynamic kernel. In view of these problems, we propose that the statistics (8) can be used to test the validity of the numerical methods used to solve the KCE in numerical cloud models, since the non-conservation of mass is an intrinsic property of the gelling-kernels after the runaway droplet is formed, and not a problem of the numerical algorithms used to integrate the KCE.

The results of the simulations presented here support the conclusion that the validity time depends on the initial spectrum and the type of collision kernel considered. For the bidisperse initial conditions used in this work, the validity time for the KCE with the hydrodynamic kernel decreases as the total concentration increases (a factor of two increase in concentration leads to a decrease from 850 to $415 \mathrm{~s}$ in the validity time) Malyshkin and Goldman (2001) showed a similar result, but for the simpler multiplicative kernels $K(x, y)=C(x y)^{\alpha}$.

\section{Discussion and conclusions}

In this paper, we evaluate the applicability of the ratio $M_{L 1, S}=S T D\left(M_{L 1}\right) / M_{L 1}$ for the largest droplet determined from realizations of the Monte Carlo method, to calculate the validity time of the KCE for the hydrodynamic kernel, which is relevant to cloud physics. This evaluation was carried out by a direct comparison with numerical solutions of the deterministic KCE. The total mass calculated from the numerical solution of the KCE starts to decrease at the moment in time

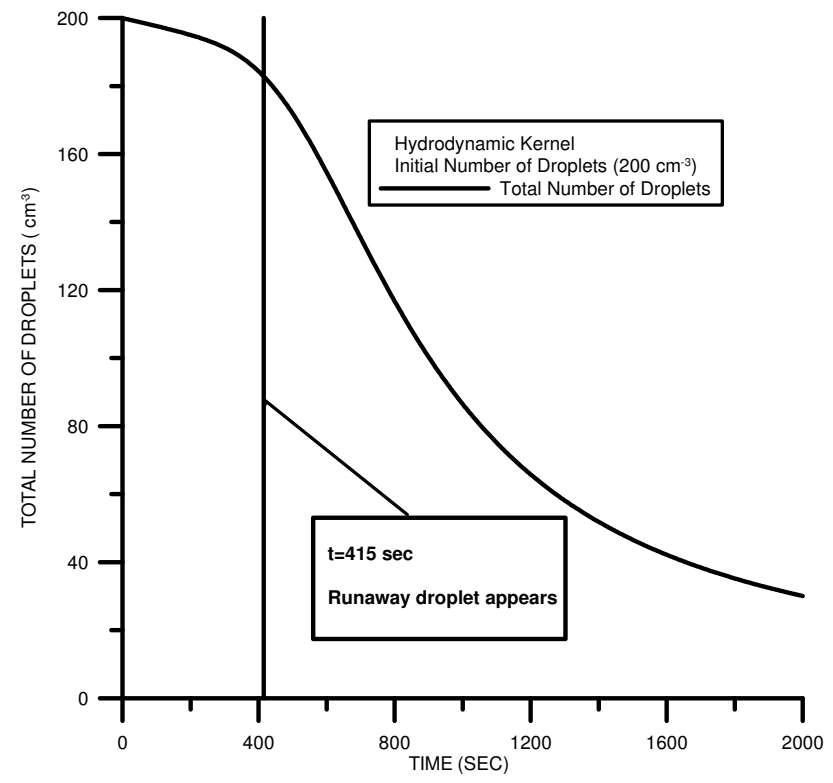

Fig. 9. Evolution of the total concentration calculated from the numerical solution of the KCE for the hydrodynamic kernel with bidisperse initial conditions. The initial total number of droplets is 200 per $\mathrm{cm}^{3}$.

when the runaway droplet forms, and the statistics $M_{L 1, S}$ obtained from Monte Carlo simulations reaches its maximum.

For the bidisperse initial conditions and the hydrodynamic kernel, the validity time decreases as the initial total droplet concentration increases, in agreement with the results obtained in other modeling studies with much simpler kernels. We confirm the fact that the KCE only describes the continuous droplet spectrum (Wetherill, 1990), and that a transfer of mass occurs from the continuous spectrum to the runaway droplet resulting in a decrease of the total mass predicted by the KCE after the maximum of $M_{L 1, S}=S T D\left(M_{L 1}\right) / M_{L 1}$ is reached. The concentration of droplets when the runaway droplet forms can be as large as $183 \mathrm{~cm}^{-3}$ (see Fig. 9), which is far larger than the threshold value of $100 \mathrm{~cm}^{-3}$ obtained by Valioulis and List (1984) in an early study of stochastic completeness of the KCE.

Another question is the possibility of existence of runaway drops, since the collisional and spontaneous breakup modes could tend to fragment them. For the simulations presented in this paper the answer is positive, since the largest drops at the start of the runaway growth have a size between 25 $40 \mu \mathrm{m}$ in radius. For example, in the simulation for the product kernel $(K(x, y)=C x y)$, at $\tau=1315 \mathrm{~s}$ the largest droplet (gel) has a radius of then $38 \mu \mathrm{m}$. For the two simulations with the hydrodynamic kernel (with initial droplet concentration of 100 and $200 \mathrm{~cm}^{-3}$ ) the runaway drop sizes were 28.5 and $36.9 \mu \mathrm{m}$ respectively, which are also smaller than the typical breakup drop size. 
The numerical criterion from the Monte Carlo realizations, of a maximum in the ratio $M_{L 1, S}=S T D\left(M_{L 1}\right) / M_{L 1}$ can also be useful to check the precision of the numerical methods to solve the KCE, since the non-conservation of mass, after the runaway droplet is formed, is an intrinsic property of the KCE (which only describes the continuum spectrum). Then, for a valid numerical scheme to solve the KCE, we aim to detect the occurrence of gelation: the decrease of the total mass after the runaway droplet is formed, together with a sudden growth of the second moment of the distribution (Eq. 2).

The results of our study are relevant to cloud modeling, since they can be used to check the reliability of the numerical methods implemented to solve the KCE, and to have estimates of the validity times of the KCE for the initial conditions under consideration.

Acknowledgements. This study was partially funded by a grant from the Consejo Nacional de Ciencia y Tecnologia de Mexico (SEP-Conacyt 62071).

Edited by: T. Garrett

\section{References}

Alfonso, L., Raga, G. B., and Baumgardner, D.: The validity of the kinetic collection equation revisited, Atmos. Chem. Phys., 8, 969-982, doi:10.5194/acp-8-969-2008, 2008.

Alfonso, L., Raga, G. B., and Baumgardner, D.: Monte Carlo simulations of two-component drop growth by stochastic coalescence, Atmos. Chem. Phys., 9, 1241-1251, doi:10.5194/acp-9-12412009, 2009.

Bayewitz, M. H., Yerushalmi, J., Katz, S., and Shinnar, R.: The extent of correlations in a stochastic coalescence process, J. Atmos. Sci., 31, 1604-1614, 1974.

Drake, R. L.: The scalar transport equation of coalescence theory: Moments and kernels, J. Atmos. Sci., 29, 537-547, 1972.
Gillespie, D. T.: A general method for numerically simulating the stochastic time evolution of coupled chemical reactions, J. Comput. Phys., 22, 403-434, 1976.

Hall, W. D.: A detailed microphysical model within a twodimensional dynamic framework: Model description and preliminary results, J. Atmos. Sci., 37, 2486-2507, 1980.

Inaba, S., Tanaka, H., Ohtsuki, K., and Nakazawa, K.: Highaccuracy statistical simulation of planetary accretion: I. Test of the accuracy by comparison with the solution to the stochastic coagulation equation, Earth Planet. Space, 51, 205-217, 1999.

Kerkweg, A., Wurzler, S., Reisin, T., and Bott, A.: On the cloud processing of aerosol particles: An entraining air-parcel model with two-dimensional spectral cloud microphysics and a new formulation of the collection kernel, Q. J.R. Meteorol. Soc., 129, $1-18,2003$.

Laurenzi, I. J., Bartels, S. L., and Diamond, S. L.: A general algorithm for exact simulation of multicomponent aggregation, J.Comput. Phys., 177, 418-449, 2002.

Long, A. B.: Solutions to the droplet collection equation for polynomial kernels, J. Atmos. Sci., 31, 1040-1051, 1974.

Malyshkin, L. and Goodman, J.: The timescale of runaway stochastic coagulation, Icarus, 50, 314-322, 2001.

Pruppacher, H. R. and Klett, J. D.: Microphysics of clouds and precipitation, Kluwer Academic Publishers, 1997.

Scott, W. T.: Analytic studies of cloud droplet coalescence, J.Atmos. Sci., 25, 54-65, 1968.

Tanaka, H. and Nakazawa, K.: Stochastic coagulation equation and the validity of the statistical coagulation equation, J. Geomag. Geoelecr., 45, 361-381, 1993.

Tanaka, H. and Nakazawa, K.: Validity of the statistical coagulation equation and runaway growth of protoplanets, Icarus, 107, 404412, 1994.

Valioulis, I. A. and List, E. J.: A numerical evaluation of the stochastic completeness of the kinetic coagulation equation, J. Atmos. Sci., 41, 2516-2529, 1984.

Wetherill, G. W.: Comparison of analytical and physical modeling of planetesimal accumulation, Icarus, 88, 336-354, 1990. 